\title{
Dynamic Knowledge Distillation with Cross-Modality Knowledge Transfer
}

\author{
Guangzhi Wang \\ School of Computing, National University of Singapore \\ Singapore \\ guangzhi.wang@u.nus.edu
}

\begin{abstract}
Supervised learning for vision tasks has achieved great success because of the advances of deep learning research in many areas, such as high quality datasets, network architectures and regularization methods. In the vanilla deep learning paradigm, training a model for visual tasks is mainly based on the provided training images and annotations. Inspired by human learning with knowledge transfer where information from multiples modalities are considered, we propose to improve visual tasks' performance by introducing explicit knowledge extracted from other modalities. As the first step, we propose to improve image classification performance by introducing linguistic knowledge as additional constraints in model learning. This knowledge is represented as a set of constraints to be jointly utilized with visual knowledge. To coordinate the training dynamic, we propose to imbue our model the ability of dynamic distilling from multiple knowledge sources. This is done via a model agnostic knowledge weighting module which guides the learning process and updates via meta-steps during training. Preliminary experiments on various benchmark datasets validate the efficacy of our method. Our code will be made publicly available to ensure reproducibility.
\end{abstract}

\section{CCS CONCEPTS}

- Computing methodologies $\rightarrow$ Image representations.

\section{KEYWORDS}

Image Classification; Knowledge Distillation

\section{ACM Reference Format:}

Guangzhi Wang. 2021. Dynamic Knowledge Distillation with Cross-Modality Knowledge Transfer. In Proceedings of the 29th ACM International Conference on Multimedia (MM '21), October 20-24, 2021, Virtual Event, China. ACM, New York, NY, USA, 5 pages. https://doi.org/10.1145/3474085.3481034

\section{INTRODUCTION}

The past decade has witnessed a great success of deep learning models in various vision tasks. Since the first success of Convolutional Networks (ConvNet) on large-scale image classification challenge [22], the deep learning models have achieved tremendous improvement with advance in model architecture, novel loss

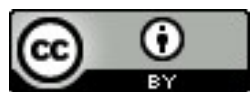

This work is licensed under a Creative Commons Attribution International 4.0 License. MM '21, October 20-24, 2021, Virtual Event, China. (C) 2021 Copyright held by the owner/author(s). ACM ISBN 978-1-4503-8651-7/21/10

https://doi.org/10.1145/3474085.3481034 functions, and availability of quality datasets. With the better regularization approaches $[6,12,23,28,29]$, those models have achieved improved results on datasets with growing scales [15, 16, 21]. From the machine learning perspective, supervised learning is a process where human knowledge gets transferred into a model in the form of annotations. For example, in image classification, such labels are explicitly describing "what can be visually perceived in the image" with an one-hot encoding. However, is it adequate for a model to learn solely from visual knowledge?

Recall that when human acquires new knowledge in a learning process, cues or instructions from multiple modalities are often involved. For example, when kids learn about the concept of husky, they may learn from its visual appearance, accompanying with its vocal behavior, the given linguistic description, and its relationship with other animals. Therefore, an intuitive approach to enhance the machine learning effective is to train models with annotations of multiple distinct modalities. However, this approach also raises several practical issues when considering the real world constraints. On one hand, it is laborious to collect extra annotations in other modalities for each training image. On the other hand, it is challenging to train the model to learn jointly from these annotated knowledge, as the modality gap between different knowledge sources may interfere the learning process. Inspired by the human learning mechanism where more abstract knowledge representations are easier to transfer than contextualized representations [3], we propose to abstract knowledge from external sources as a set of distance constraints.

In this work, we propose to utilize external knowledge, especially knowledge with semantic information, to mine a set of relations between concepts equipped with a meta-learning strategy to incorporate these knowledge into the learning system. Our proposed method is compatible with various external knowledge sources as along as the relations between concepts can be explicitly or implicitly extracted from it. To incorporate these knowledge in the machine learning process, we represent these knowledge in the form of a set of distance constraints, so as to encourage the features extracted from images to follow the concept relationships provided in external knowledge. With these constraints, the external knowledge is represented in a model agnostic way that can be easily incorporated into the training process and jointly learned with visual knowledge. As is shown in [4], in general, visual similarity grows with semantic similarity but they are not perfectly aligned, suggesting visual and semantic knowledge are able to cooperate and complement each other.

Due to the existence of semantic gap between different modalities, simply introducing these knowledge into a visual learning model may lead to sub-optimal or even deteriorate performance. 
Besides, the constantly changing model dynamics also brings up difficulties of absorbing external knowledge. To this end, we propose a novel meta-learning strategy which learns to transfer external knowledge into the learning system by giving the importance of external knowledge. We employ a meta-learner which takes an abstract of model state at each time step as input and output the importance of external knowledge. To coordinate with the sequential nature of a learning process, we follow [2] to model the metalearner as a Long Short Term Memory (LSTM) network [14]. With the meta-learner, our model is also learning "how to learn" from multiple knowledge sources, which is better aligned with human's learning process.

The main contributions of this paper are as follows:

- Besides visual knowledge, we propose to further improve image classification performance by incorporating external knowledge into our learning framework. We represent external knowledge as a set of distance constraints to help the absorbing of external knowledge.

- We propose to enable our model the same ability via metalearning strategy which learns how to combine external knowledge into current model according to model state by predicting the importance of external knowledge.

- We perform preliminary experiments on multiple benchmark datasets. The results and statistical evidence demonstrate the efficacy of the proposed method.

\section{BACKGRAOUND AND RELATED WORK}

\subsection{Semantically Informed Learning}

In computer vision literature, some works utilize semantic information from linguistic sources to assist the learning of vision recognition. Zero-shot Learning (ZSL) aims to recognize novel classes with no training instances by transferring knowledge from source classes [7]. It was first explored by using attribute-based semantic annotations, which is expensive for large-scale dataset [7, 9]. Then, a series of work $[1,8,11,20]$ utilize semantic word vectors as a type of semantic representations, which reduce the expensive human annotation. Later work in ZSL proposed to learn a direct mapping from visual representations to semantic representations [10], which is then used for recognizing unseen classes. Our work also takes advantage of concept information in linguistic domain to augment the visual learning process. Instead of directly employing semantic representations, our work employs the concept relations which is contained or implied in various knowledge sources.

\subsection{Vision-Language pre-training}

With the success of Transformer [27], vision-language pre-training is rapidly growing recently. Existing methods employ BERT-like [5] objective to pre-train a transformer-like model so as to obtain the joint representation of both modalities. These pre-trained models are later used for downstream tasks such as visual questionanswering, image-captioning and image-text retrieval etc. [1719, 24-26]. In contrast, our work represents the knowledge from another modality as a set of constraints which is learned with the help of a meta-learner.

\section{PROBLEM DEFINITION}

In this paper, we consider the problem of applying external knowledge to the image classification task in a closed-world assumption of $C$ categories $C=\left\{c_{1}, c_{2}, \ldots, c_{C}\right\}$. There is a set of i.i.d. training data $D=\left\{\left(X_{1}, y_{1}\right),\left(X_{2}, y_{2}\right), \ldots,\left(X_{N}, y_{N}\right)\right\}$, where $X_{i}$ denotes the $i$ - th image, $y_{i} \in C$ is the ground-truth label of $X_{i}$ and $N$ is the total number of training images. In addition, there is a knowledge source $K$ which provides extra relational information about the categories. The classification model consists of a visual feature extractor $g(\cdot)$ and a linear classifier combined with a softmax layer $f(\cdot)$. The $l$-dimensional visual feature of the $\mathrm{u}$-th image is extractedas $x_{i}=g\left(X_{i}\right)$ and fed into the classifier for the final class scores $p_{i}=f\left(g\left(X_{i}\right)\right)$.

The incorporation of external knowledge during model training requires setting up a proper connection between $D$ and $K$. While it is difficult to directly connect the semantics of a category with its visual representation due to the semantic gap, it is plausible to transfer the similarities in different spaces. In other words, we assume that the semanticaly similar categories also hae similar visual features. Starting from this assumption, we further suume that $K$ can be presented as a function $K \in C \times C \longrightarrow R$, in which $K\left(c_{i}, C_{j}\right)$ is the category similarity score between two categories $c_{i}$ and $c_{j}$, and we declare $V \in R^{l} \times R^{l} \longrightarrow R$ which measures the visual similarity between two visual features. The connection between $D$ and $K$ can be simply described as: the visual similarities should be aligned with the category similarities. Formally, the training objective is

$$
\max \frac{1}{N} \sum_{i=1}^{N} y_{i} \log p_{i}+\frac{1}{N^{2}} \sum_{i=1}^{N} \sum_{j=1}^{N} h\left(V\left(x_{i}, x_{j}\right), K\left(c_{i}, c_{j}\right),\right.
$$

where $h \in R \times R \longrightarrow R$ measures the consistency between the visual similarity and category similarity.

\section{METHODOLOGY}

\subsection{Overview}

In accordance with the training objective in Equation (1), we propose the Knowledge Alignment Network. The model employs a vanilla backbone network designed for image classification task, which is updated using an anchor training sample $X_{a}$ and a regular classification loss $\mathcal{L}_{c l s}$. At the same time, we propose to learning the model with the guidance of category similarity $K$ extracted from external knowledge. Specifically, together with the anchor image $X_{a}$, we sample another three images $X_{p}, X_{w n}$ and $X_{s n}$ of three distinct categories: positive, weak negative and strong negative, respectively. We then compute three knowledge transferring losses in term of visual features and the similarities between sampled categories. These knowledge-guided losses, along with the classification loss $\mathcal{L}_{c l s}$, are fed into the meta-learner, which outputs the mixing coefficients for each loss terms using the context provided by $\mathcal{L}_{c l s}$. The full optimization objective $\mathcal{L}_{\text {full }}$ is the sum of $\mathcal{L}_{c l s}$ and the weighted combination of the knowledge transferring loss $\hat{\mathcal{L}}_{K G}$. 


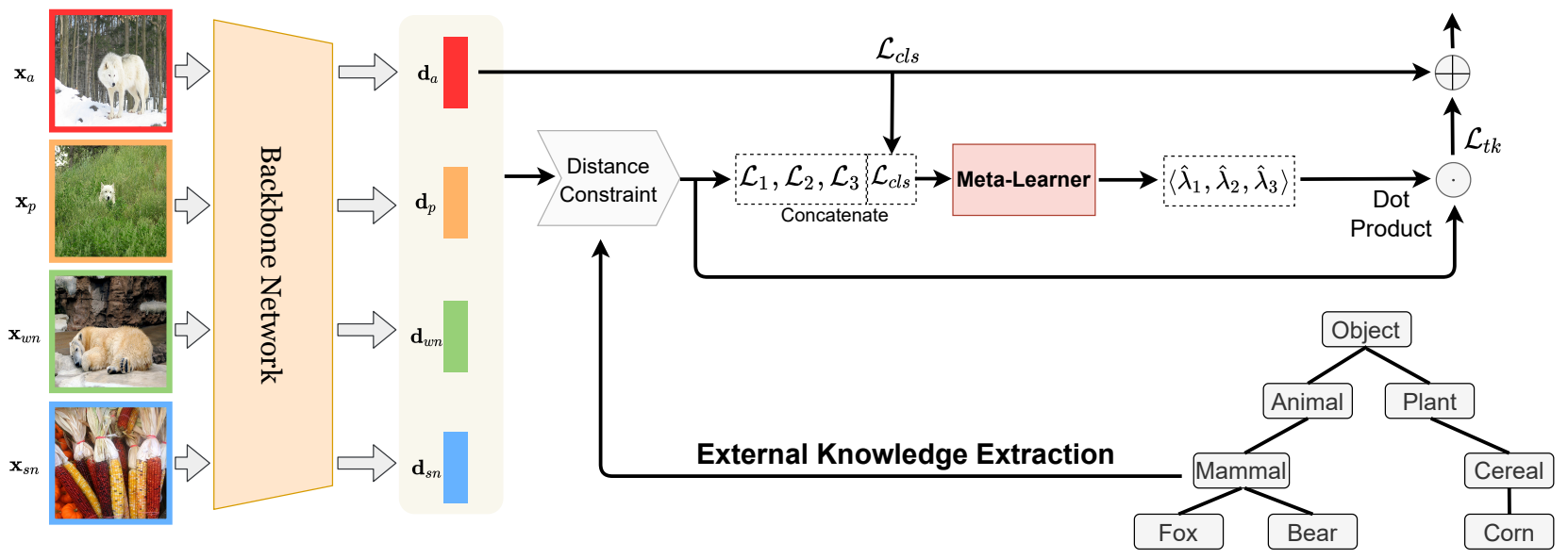

Figure 1: Overview of the proposed knowledge guided learning framework. For each given sample $x_{a}$, we sample 3 more samples, namely positive $x_{p}$, weak negative $\mathrm{x}_{w n}$ and strong negative $x_{s n}$, which are then forwarded to a ConvNet to extract their features. We calculate 3 triplet losses $\mathcal{L}_{1}, \mathcal{L}_{2}$ and $\mathcal{L}_{3}$ Using the feature of the four samples, and a classification loss using only the anchor feature $d_{a}$ and its ground-truth label. Then $\mathcal{L}_{c l s}$ is then concatenated with $\left\langle\mathcal{L}_{1}, \mathcal{L}_{2}, \mathcal{L}_{3}\right\rangle$ to be the input of the meta-learner to predict the weights of each distance constraint. $\mathcal{L}_{t k}$ is calculated as the dot product between the triplet losses and the predicted weights. Finally, the model is trained by the sum of $\mathcal{L}_{c l s}$ and $\hat{\mathcal{L}}_{t k}$.

\subsection{External Knowledge Transfer}

This section introduces the definition of the novel knowledgetransferring losses. Recall that our goal is to align the visual similarities with the reference category similarities. Towards this goal, we first sample three images, $X_{p}, X_{w n}, X_{s w}$, for each anchor image $X_{a}$, such that $c_{a}=c_{p}$ and $K\left(c_{a}, c_{p}\right)>K\left(c_{a}, c_{w} n\right)>K\left(c_{a}, c_{s n} . K(\cdot, \cdot)\right.$ is the category similarity. Then, we apply the following constraints on the corresponding visual features:

$$
\begin{gathered}
V\left(x_{a}, x_{p}\right)-V\left(x_{a}, x_{s n}\right)>m\left(c_{a}, c_{p}, c_{s n}\right) \\
V\left(x_{a}, x_{p}\right)-V\left(x_{a}, x_{w n}\right)>m\left(c_{a}, c_{p}, c_{w n}\right) \\
V\left(x_{a}, x_{w n}\right)-V\left(x_{a}, x_{s n}\right)>m\left(c_{a}, c_{w n}, c_{s n}\right),
\end{gathered}
$$

where $V(\cdot, \cdot)$ measures the cosine similarity and $m(\cdot, \cdot, \cdot)$ is a margin value related to the samples' categories and is defined as follows:

$$
m\left(c_{1}, c_{2}, c_{3}\right)=\tanh \left(\beta \cdot \log \frac{K\left(c_{1}, c_{2}\right)}{K\left(c_{1}, c_{3}\right)}\right) .
$$

During training, we impose the constraints as a set of triplet loss terms:

$$
\begin{aligned}
& \mathcal{L}_{1}=\sum_{i=1}^{N}\left[m_{p(i), s n(i)}-V\left(x_{a(i)}, x_{p(i)}\right)+s\left(x_{a(i)}, x_{s n(i)}\right)\right]_{+} \\
& \mathcal{L}_{2}=\sum_{i=1}^{N}\left[m_{p(i), w n(i)}-V\left(x_{a(i)}, x_{p(i)}\right)+V\left(x_{a(i)}, x_{w n(i)}\right)\right]_{+} \\
& \mathcal{L}_{3}=\sum_{i=1}^{N}\left[m_{w n(i), s n(i)}-V\left(x_{a(i)}, x_{w n}\right)+V\left(x_{a(i)}, x_{s n(i)}\right)\right]_{+}
\end{aligned}
$$

where $[\cdot]_{+}$represents $\max (0, \cdot) \cdot x_{p}(i), x_{w n(i)}$ and $x_{s n(i)}$ respectively represents the features of the sampled positive, weak negative and strong negative image for the $i-$ th anchor. $m_{\mathcal{u}, v}$ indicates $m\left(c_{a}, c_{u}, c_{v}\right)$. The overall losses for transferring knowledge is calculated as

$$
L_{t k}=\lambda_{1} \mathcal{L}_{1}+\lambda_{2} \mathcal{L}_{2}+\lambda_{3} \mathcal{L}_{3},
$$

where $\lambda_{*}$ are the weighting coefficients that decide the importance of external knowledge introduced by each loss term. The weighting coefficients can be determined through grid search.

\subsection{Learning to Learn with External Knowledge}

So far, we have introduced the basic framework that introduces knowledge transferring losses to the training process. However, the proper moment and degree to incorporate these losses remains unclear. Simply combining these loss terms with a fixed weight or hand-crafted weight scheduling as in conventional methods could lead to sub-optimal performance due to the complexity of training dynamics. Therefore, we propose a knowledge weighting module, which learns to guide the learning process by weighting each knowledge transferring loss term dynamically.

Given the sequential nature of a model training procedure, we implement the meta-learner as an LSTM model to better capture the occurred learning dynamics. At each time step, the LSTM takes the concatenation of all loss values as input and outputs the knowledge mixing coefficients $\hat{\lambda}_{*}$. The loss values can be viewed as a high-level abstraction of model states.

The meta-learner works as follows:

$$
\begin{gathered}
I=\mathbf{W}\left[\mathcal{L}_{c l s} ; \mathcal{L}_{1} ; \mathcal{L}_{2} ; \mathcal{L}_{3}\right] \\
h_{t}, \hat{O}=\operatorname{LSTM}\left(I, h_{t-1}\right) \\
\hat{\lambda}_{1}, \hat{\lambda}_{2}, \hat{\lambda}_{3}=\gamma \cdot \operatorname{sigmoid}(\hat{O}),
\end{gathered}
$$

where [;] means concatenation, $\mathbf{W}$ is a learn-able matrix, and $\gamma$ is a hyper-parameter to bound the range of the weights. Here we use $\operatorname{sigmoid}(\cdot)$ to bound the range of the weight values. 
Table 1: Classification Performance on CIFAR-10. The results are reported as mean \pm std from 3 runs. ${ }^{*}$ and ${ }^{* *}$ indicate the p-value is less than 0.05 and 0.01 , respectively.

\begin{tabular}{l|c}
\hline Method & Accuracy (\%) \\
\hline ResNet-18 [6] & $95.28 \pm 0.21$ \\
ResNet-18 (reproduced) & $95.14 \pm 0.03$ \\
ResNet-18 + Ours (WordNet) & $95.47 \pm 0.06^{* *}$ \\
ResNet-18 + Ours (Glove) & $\mathbf{9 5 . 4 8} \pm 0.08^{* *}$ \\
\hline
\end{tabular}

Given these predicted weight values, we calculate the weighted and knowledge-guided loss term as follows:

$$
\hat{\mathcal{L}}_{t k}=\hat{\lambda}_{1} \mathcal{L}_{1}+\hat{\lambda}_{2} \mathcal{L}_{2}+\hat{\lambda}_{3} \mathcal{L}_{3}
$$

Finally, the backbone network is updated with the full optimization objective:

$$
\mathcal{L}_{\text {full }}=\mathcal{L}_{c l s}+\hat{\mathcal{L}}_{t k}
$$

where $\mathcal{L}_{c l s}$ is the cross entropy loss for classification:

$$
\mathcal{L}_{c l s}=-\frac{1}{N} \sum_{i=1}^{N} y_{i} \cdot \log \left(f\left(g\left(x_{i}\right)\right)\right)
$$

\section{EXPERIMENTS}

\subsection{Dataset}

CIFAR-10/100 [15] CIFAR-10 and CIFAR-100 are two datasets of tiny images with $32 \times 32$ pixels. Both datasets consist of 50,000 images for training and 10,000 images for test. The two datasets have 10 and 100 classes, respectively.

Tiny ImageNet is a subset of ImageNet with a smaller training set of 100,000 images and a validation set of 10,000 images in 200 classes.

ImageNet [21] is one of largest publicly available dataset for image classification, which has a training set of 1.3 million hand-annotated images and a validation set of 50,000 images. There are totally 1,000 classes and we resize the images into size of $224 \times 224$ in all our experiments.

\subsection{Implementation Details}

On CIFAR-10/100, we carry out experiments on ResNet-18. While on Tint-ImageNet and ImageNet, we carry out experiments on ResNet-50. We train the models with an initial learning rate of 0.1 for 200 epochs. The learning rate is multiplied by 0.2 at 60 th, 120 th and 160th epoch. For all models, We use SGD optimizer with a momentum of 0.9 and weight decay of $5 \mathrm{e}-4$.

\subsection{Experimental Result}

To validate the efficacy of our method, we experiment our method on benchmarks. In the following tables, all reported digits are are averaged over 3 independent runs with different random seed. All results are reported as mean \pm std.

On CIFAR-10, we observe consistent improvements of $0.31 \%$, $0.32 \%$. Similarly, on CIFAR-100, we obtain an improvement of $1.02 \%$ with WordNet and $1.10 \%$ with GloVe. The difference of improvement may be attributed to the number of classes in a dataset, which is an important factor of the proposed method. On Tiny-ImageNet,
Table 2: Classification Performance on CIFAR-100. width $=1.0$

\begin{tabular}{l|l}
\hline Method & Accuracy (\%) \\
\hline ResNet-18 [6] & $77.54 \pm 0.31$ \\
ResNet-18 (reproduced) & $77.24 \pm 0.20$ \\
ResNet-18 + Ours (WordNet) & $78.26 \pm 0.30^{*}$ \\
ResNet-18 + Ours (Glove) & $\mathbf{7 8 . 3 4} \pm 0.18^{* *}$ \\
\hline
\end{tabular}

Table 3: Classification performance on Tiny ImageNet.

\begin{tabular}{l|c}
\hline Method & Accuracy $(\%)$ \\
\hline ResNet-50 (reproduced) & $79.30 \pm 0.09$ \\
ResNet-50 + Ours (WordNet) & $\mathbf{7 9 . 8 4} \pm 0.05$ \\
ResNet-50 + Ours (Glove) & $79.81 \pm 0.02$ \\
\hline
\end{tabular}

Table 4: Classification Performance on ImageNet.

\begin{tabular}{l|c|c}
\multicolumn{3}{c}{ width=1.0 } \\
\hline Method & Top 1 (\%) & Top 5 (\%) \\
\hline ResNet-50 [13] & 76.00 & 93.00 \\
ResNet-50 (reproduced) & 76.13 & 92.86 \\
ResNet-50 + Ours (WordNet) & $\mathbf{7 6 . 6 8}$ & $\mathbf{9 3 . 1 0}$ \\
ResNet-50 + Ours (Glove) & 76.59 & 93.08 \\
\hline
\end{tabular}

We show our results in Table 3. On ResNet-50, we observe an improvement of $0.54 \%$ with WordNet as tacit knowledge source and $0.51 \%$ with GloVe, which shows different improvements when the scale of dataset varies. Lastly, on ImageNet, we reach an improvement of $0.55 \%$ on Top- 1 accuracy and $0.24 \%$ on Top- 5 accuracy with WordNet and similar improvements with Glove. These results validate the efficacy of our method with datasets of various scales and with both external knowledge sources.

\section{DISCUSSION}

In this paper, we propose a novel learning paradigm which introduces external knowledge into a visual learning system. Also we propose a meta-learning strategy to better utilize these external knowledge into the model training process. Extensive experimental results and ablation studies validated the efficacy and necessity of each component in our proposed method under various benchmark datasets and backbones. We believe such learning paradigm can be potentially extended to vision tasks other than classification, as long as the relationship between concepts can be modeled explicitly or implicitly. For example, in action recognition, we may introduce the similarity between actions provided in external knowledge sources with similar strategy. Besides, external knowledge from other sources, such as other types of knowledge graphs, representations from a pretrained-model model can also be taken as external knowledge source to augment model training. On the other hand, it is also possible to enhance linguistic knowledge learning by employing visual knowledge.

\section{REFERENCES}

[1] Zeynep Akata, Scott Reed, Daniel Walter, Honglak Lee, and Bernt Schiele. 2015. Evaluation of Output Embeddings for Fine-grained Image Classification. In CVPR. 2927-2936. 
[2] Marcin Andrychowicz, Misha Denil, Sergio Gomez, Matthew W Hoffman, David Pfau, Tom Schaul, Brendan Shillingford, and Nando De Freitas. 2016. Learning to Learn by Gradient Descent by Gradient Descent. In NIPS. 3981-3989.

[3] National Research Council. 2000. How People Learn: Brain, Mind, Experience, and School: Expanded Edition. The National Academies Press.

[4] Thomas Deselaers and Vittorio Ferrari. 2011. Visual and Semantic Similarity in ImageNet. In CVPR. 1777-1784

[5] Jacob Devlin, Ming-Wei Chang, Kenton Lee, and Kristina Toutanova. 2019. BERT: Pre-training of Deep Bidirectional Transformers for Language Understanding. In NAACL-HLT. 4171-4186.

[6] Terrance DeVries and Graham W Taylor. 2017. Improved Regularization of Convolutional Neural Networks with Cutout. arXiv preprint arXiv:1708.04552 (2017).

[7] Ali Farhadi, Ian Endres, Derek Hoiem, and David Forsyth. 2009. Describing Objects by Their Attributes. In CVPR. 1778-1785.

[8] Andrea Frome, Greg S Corrado, Jon Shlens, Samy Bengio, Jeff Dean, Marc'Aurelio Ranzato, and Tomas Mikolov. 2013. DeViSE: A Deep Visual-Semantic Embedding Model. In NIPS. 2121-2129.

[9] Yanwei Fu, Timothy M Hospedales, Tao Xiang, and Shaogang Gong. 2015. Transductive Multi-view Zero-shot Learning. IEEE Transactions on Pattern Analysis and Machine Intelligence 37, 11 (2015), 2332-2345.

[10] Yanwei Fu and Leonid Sigal. 2016. Semi-Supervised Vocabulary-Informed Learning. In CVPR. 5337-5346.

[11] Zhenyong Fu, Tao Xiang, Elyor Kodirov, and Shaogang Gong. 2015. Zero-shot Object Recognition by Semantic Manifold Distance. In CVPR. 2635-2644.

[12] Golnaz Ghiasi, Tsung-Yi Lin, and Quoc V Le. 2018. DropBlock: A regularization method for convolutional networks. In NeurIPS. 10727-10737.

[13] Kaiming He, Xiangyu Zhang, Shaoqing Ren, and Jian Sun. 2016. Deep Residual Learning for Image Recognition. In CVPR. 770-778.

[14] Sepp Hochreiter and Jürgen Schmidhuber. 1997. Long Short-Term Memory. Neural Computation 9, 8 (1997), 1735-1780.

[15] Alex Krizhevsky. 2009. Learning Multiple Layers of Features from Tiny Images. Technical Report. University of Toronto.

[16] Yann LeCun, Léon Bottou, Yoshua Bengio, and Patrick Haffner. 1998. Gradientbased Learning Applied to Document Recognition. Proc. IEEE 86, 11 (1998), $2278-2324$
[17] Jiasen Lu, Dhruv Batra, Devi Parikh, and Stefan Lee. 2019. ViLBERT: Pretraining Task-agnostic Visiolinguistic Representations for Vision-and-Language Tasks. In NeurIPS. 13-23.

[18] Jiasen Lu, Vedanuj Goswami, Marcus Rohrbach, Devi Parikh, and Stefan Lee. 2020. 12-in-1: Multi-Task Vision and Language Representation Learning. In CVPR. 10437-10446.

[19] Duy-Kien Nguyen and Takayuki Okatani. 2019. Multi-task Learning of Hierarchical Vision-Language Representation. In CVPR. 10492-10501.

[20] Mohammad Norouzi, Tomas Mikolov, Samy Bengio, Yoram Singer, Jonathon Shlens, Andrea Frome, Greg S Corrado, and Jeffrey Dean. 2014. Zero-shot Learning by Convex Combination of Semantic Embeddings. In ICLR.

[21] Olga Russakovsky, Jia Deng, Hao Su, Jonathan Krause, Sanjeev Satheesh, Sean Ma, Zhiheng Huang, Andrej Karpathy, Aditya Khosla, Michael Bernstein, et al. 2015. Imagenet Large Scale Visual Recognition Challenge. International journal of computer vision 115, 3 (2015), 211-252.

[22] Karen Simonyan and Andrew Zisserman. 2015. Very deep convolutional networks for large-scale image recognition. In ICLR.

[23] Nitish Srivastava, Geoffrey Hinton, Alex Krizhevsky, Ilya Sutskever, and Ruslan Salakhutdinov. 2014. Dropout: A Simple Way to Prevent Neural Networks from Overfitting. The fournal of Machine Learning Research 15, 1 (2014), 1929-1958.

[24] Weijie Su, Xizhou Zhu, Yue Cao, Bin Li, Lewei Lu, Furu Wei, and Jifeng Dai. 2020. VL-BERT: Pre-training of Generic Visual-Linguistic Representations. In ICLR

[25] Chen Sun, Austin Myers, Carl Vondrick, Kevin Murphy, and Cordelia Schmid. 2019. VideoBERT: A Joint Model for Video and Language Representation Learning. In ICCV. 7464-7473.

[26] Hao Tan and Mohit Bansal. 2019. LXMERT: Learning Cross-Modality Encoder Representations from Transformers. In EMNLP. 5103-5114

[27] Ashish Vaswani, Noam Shazeer, Niki Parmar, Jakob Uszkoreit, Llion Jones, Aidan N Gomez, Łukasz Kaiser, and Illia Polosukhin. 2017. Attention is All You Need. In NIPS. 6000-6010.

[28] Li Wan, Matthew D. Zeiler, Sixin Zhang, Yann LeCun, and Rob Fergus. 2013. Regularization of Neural Networks using DropConnect. In ICML (FMLR Workshop and Conference Proceedings, Vol. 28). 1058-1066.

[29] Yulin Wang, Xuran Pan, Shiji Song, Hong Zhang, Cheng Wu, and Gao Huang. 2019. Implicit Semantic Data Augmentation for Deep Networks. In NeurIPS. 12614-12623. 